\title{
Various results in relation with the hypergeometric equations and the hypergeometric functions in the complex plane
}

\author{
Hüseyin Irmak
}

\begin{abstract}
The main purpose of this investigation is to specify an extensive relation between the hypergeometric functions and the hypergeometric equations in the complex plane and then to point various implications of our main result, conclusion and also recommendations out.
\end{abstract}

Mathematics Subject Classification (2010): 30A10, 34A40, 33C05, 33C20, 33C45, 30C15, 33D15, 30D05, 33E20, 34K06, 37K20.

Keywords: Complex plane, the second-order ordinary linear differential equation, the confluent hypergeometric equation, the confluent hypergeometric function, special functions defined by complex series, inequalities and equations in the complex plane.

\section{Introduction, definitions and motivation}

In this section, we first introduce the (Gauss) hypergeometric function, the (Gauss) hypergeometric differential equation and certain well-known relationship between them. We then focus on revealing some possible interesting results associating with certain novel and extensive relationships between them. In the light of the alleged results, we also remark that there are a number of important implications of our results between various (differential) equations and special functions in the complex plane.

The well-known hypergeometric functions (and also the hypergeometric differential equations) with complex (or real) variable have been attracting much more attention in the literature. This interest is due to its importance as solutions (or applications) of many applied problems in mathematics given by the references in [1]-[3], [8], [7], [12], [13], [14], [18], [30], [38], [39], [44]-[45] and [49], in Statistics and Probability given by [2], [11], [14], [21], [33] and [38], in physics [2], [3], [7], [11], [19], 
[25] and [38], and also in the majority of engineering sciences given by [2], [5], [7], [9], [14], [17], [22], [24], [25], [33], [34], [38], [40], [43], [48], [50] and [51].

As is known, the hypergeometric functions (and also the functions being the solutions of hypergeometric differential equations) constitute a wide and important class of special functions with complex (or real) variable. In particularly, a great number of special functions of mathematical physics turn out to be hypergeometric function. In addition, multivariate hypergeometric functions can be introduced as solutions to certain overdetermined systems of linear partial differential equations with polynomial coefficients. In general, such systems of equations are of substantial independent interest and appear in several applications. The simplest ordinary differential equation of this kind is the Gauss hypergeometric equation in the literature. Any second-order linear differential equation with three regular singularities in the Riemann sphere can be also reduced to the Gauss equation by the help of a suitable change of the variables. For their details, it can be also checked the works given in [2], [5]-[6], [9], [10], [16], [17], [20], [23], [31], [32], [38]-[37], [41], [43]-[48], [50] and [51].

Since our main purpose in this scientific work is to reveal certain novel and/or non-linear relationships between the (Gauss, Gaussian or ordinary) hypergeometric functions and certain special functions in the complex plane, primarily, we have to remember certain basic information about the functions and the (differential) equations which are related to the mentioned topics and the related ones. In mathematics, we note that the hypergeometric function is a special function represented by series (or integral), which includes many other special functions as specific or limiting cases. This function is a solution of a second-order linear ordinary differential equation that every second-order linear ordinary differential equation with three singular points can be transformed into the related differential equation. For their details, let us now start by recalling (or introducing) the following information.

First of them, here and throughout this present work, firstly, we note that the well-known notations:

$$
\mathbb{N} \quad, \quad \mathbb{Z}^{-}, \quad \mathbb{R} \quad, \quad \mathbb{C} \text { and } \mathbb{U}
$$

denote the set of natural numbers, the set of negative integers, the set of real numbers, the set of complex numbers and the open unit disk in the complex plane, respectively.

The first important topic is related to the function set by the series, so let me know about it now. For this, the following functional series in the complex variable $z$, called the (Gauss, Gausian or ordinary) hypergeometric function, is denoted by any one of the notations: ${ }_{2} F_{1}(\alpha, \beta ; \gamma ; z), F(\alpha, \beta ; \gamma ; z)$ and $\mathbf{F}(\alpha, \beta ; \gamma ; z)$ and also defined by

$$
\begin{aligned}
F(\alpha, \beta ; \gamma ; z) & =1+\frac{\alpha \beta}{\gamma} z+\frac{\alpha(\alpha+1) \beta(\beta+1)}{\gamma(\gamma+1)} \frac{z^{2}}{2 !} \\
& +\frac{\alpha(\alpha+1)(\alpha+2) \beta(\beta+1)(\beta+2)}{\gamma(\gamma+1)(\gamma+2)} \frac{z^{3}}{3 !}+\cdots \\
& =\sum_{n=0}^{\infty} \frac{(\alpha)_{n}(\beta)_{n}}{(\gamma)_{n}} \frac{z^{n}}{n !}
\end{aligned}
$$


where $\alpha \in \mathbb{C}-\mathbb{Z}_{0}^{-}, \beta \in \mathbb{C}-\mathbb{Z}_{0}^{-}, \gamma \in \mathbb{C}-\mathbb{Z}_{0}^{-}$and $z \in \mathbb{C}$, and, in terms of the Gamma function $\Gamma(z)$, the (rising) Pochhammer symbol, i.e., the symbol $(v)_{n}$ is also defined by

$$
\begin{aligned}
(v)_{n} & = \begin{cases}1 & (n=0) \\
v(v+1) \cdots(v+n-1) & (n \in \mathbb{N})\end{cases} \\
& =\frac{\Gamma(v+n)}{\Gamma(v)},
\end{aligned}
$$

where $n \in \mathbb{N}_{0}:=\mathbb{N} \cup\{0\}$ and $v \in \mathbb{C}-\mathbb{Z}_{0}^{-}$.

We here note that, as certain characteristic properties of the series in (1.1) together with (1.2), it is absolutely and uniformly convergent in the disk $\mathbb{U}$. The convergence also extends over the unit circle when $\Re e(\alpha+\beta-\gamma)<0$, it converges at all the points of the unit circle except the point $z=1$ when $0 \leq \Re e(\alpha+\beta-\gamma)<1$. Nevertheless, there exists an analytic continuation of the hypergeometric function in (1.1) to the exterior $|z|>1$ of the unit disk with the slit $(1, \infty)$. The function defined by the series in (1.1), namely, $F(\alpha, \beta ; \gamma ; z)$ is an univalent-analytic function in the complex plane with slit $(1, \infty)$. When $\alpha$ or $\beta$ are zero or negative integers, the series given (1.1) terminates after a finite number terms and the hypergeometric function is a polynomial in $z$. Further, when $n \in \mathbb{Z}_{0}^{-}$, the function given by (1.1) is not defined but the limit can be considered there.

It follows from (1.1) that

$$
F(\alpha, \beta ; \gamma ; z)=F(\beta, \alpha ; \gamma ; z)
$$

and, in the light of the identity (1.2), it is easily shown that

$$
\frac{d}{d z}(F(\alpha, \beta ; \gamma ; z))=\frac{\alpha \beta}{\gamma} F(\alpha, \beta ; \gamma ; z)
$$

and, more generally,

$$
\frac{d^{n}}{d z^{n}}(F(\alpha, \beta ; \gamma ; z))=\frac{(\alpha)_{n}(\beta)_{n}}{(\gamma)_{n}} F(\alpha+n, \beta+n ; \gamma+n ; z)
$$

for all $n \in \mathbb{N}_{0}$. In particular, we note that the properties relating to the derivative given in (1.4) (or (1.5) will be useful in the simpler expression of complex statements for equations (or inequalities) stated by derivative(s).

The second important issue is associated with a homogenous differential equation in the complex plane. So, there is a need to present about it. For this, the function, given by the series in the form (1.1), is a solution of the following homogeneous differential equation given by:

$$
z(1-z) \frac{d^{2} w}{d z^{2}}+[\gamma-(\alpha+\beta+1) z] \frac{d w}{d z}-\alpha \beta w(z)=0,
$$

where $\alpha \in \mathbb{C}-\mathbb{Z}_{0}^{-}, \beta \in \mathbb{C}-\mathbb{Z}_{0}^{-}, \gamma \in \mathbb{C}-\mathbb{Z}_{0}^{-}$and $z \in \mathbb{C}-[1, \infty)$.

The differential equation just above is also known as the (Euler's) hypergeometric differential equation in the literature. By a simple focusing, it can be easy seen that, for the equation in (1.6), there have three singular points, which are 0,1 and $\infty$. 
Because of the important relation between the function given by (1.1) and the equation (1.6), there are a large number of the possible special functions specified by the function given (1.1) and, naturally, they will also include many significant relations with the differential equation in (1.6). We can now continue to determine those relations (or properties) in the second section.

\section{Main result, comment and recommendations}

As is known, the proof technique used in the proof of the theories is very important in the theoretical studies. A few examples given as in [27]-[29] are some of the proofs used in the complex functions theory, which is appropriate for the purpose of this study. As a different proof method will be used in this paper, in order to prove our main result, initially, we need to recall the following-well-known assertion (see [35] and [36]).

Lemma 2.1. Let a function $p(z)$ in the form:

$$
p(z)=1+e_{n} z^{n}+e_{n+1} z^{n+1}+e_{n+2} z^{n+2}+\cdots
$$

be analytic in the open set:

$$
\mathbb{U}=\{z: z \in \mathbb{C} \text { and }|z|<1\},
$$

where $n \in \mathbb{N}$ and $e_{n} \in \mathbb{C}$. such that

If the function $p(z)$ is not with positive real part in $\mathbb{U}$, then there is a point $z_{0} \in \mathbb{U}$

$$
\left.p(z)\right|_{z=z_{0}}=i \lambda \quad \text { and }\left.\quad z \frac{d}{d z}(p(z))\right|_{z=z_{0}}=\mu
$$

where

$$
\lambda \in \mathbb{R}-\{0\} \quad, \quad \mu \in \mathbb{R} \quad \text { and } \quad \mu \leq-n \frac{1+\lambda^{2}}{2} \quad(n \in \mathbb{N}) .
$$

Let us now introduce a third-order differential equations with (complex) variable coefficients in the complex plane, which will play an important role in our main result, as in the following form:

$$
\begin{aligned}
(1-z) z^{2} \frac{d^{3} \omega}{d z^{3}} & +z[1+\gamma-(3+\alpha+\beta) z] \frac{d^{2} \omega}{d z^{2}} \\
& -(1+\alpha+\beta) z \frac{d \omega}{d z}=\alpha \beta \Phi(z),
\end{aligned}
$$

where $z \in \mathbb{U}, \alpha \in \mathbb{C}-\mathbb{Z}_{0}^{-}, \beta \in \mathbb{C}-\mathbb{Z}_{0}^{-}$and $\gamma \in \mathbb{C}-\mathbb{Z}_{0}^{-}$. In special, note that, since the differential equation in (2.4) is the derivative of both sides of the equation in (1.6), clearly, both the function $w:=F(\alpha, \beta ; \gamma ; z)$ is the solution for the (complex) differential equation in (2.4) and the (complex) function $\Phi(z)$ is analytic in $\mathbb{U}$.

Theorem 2.2. Let the functions $\omega:=\omega(z)$ and $\Phi(z)$ be in the forms defined by (1.1) and (2.4), respectively. For any $z \in \mathbb{U}$ and for some $\alpha \in \mathbb{C}-\mathbb{Z}_{0}^{-}$and $\beta \in \mathbb{C}-\mathbb{Z}_{0}^{-}$, if 
any one of the cases of the following inequality:

$$
\Re e(\alpha \beta \Phi(z))\left\{\begin{array}{lll}
>-\frac{\Re e(\alpha \beta)}{2} & \text { if } & \Re e(\alpha \beta) \geq 0 \\
<-\frac{\Re e(\alpha \beta)}{2} & \text { if } \quad \Re e(\alpha \beta) \leq 0
\end{array},\right.
$$

is satisfied, then

is also satisfied

$$
\Re e(\omega(z))>0 \quad(z \in \mathbb{U})
$$

Proof. Since the function $\omega \equiv \omega(z)$ has the form given by (1.1), it is a particular solution for the differential equation given by (1.6). By taking into account this fact, let us take $p(z)$ as

$$
p(z)=\omega(z)(\equiv F(\alpha, \beta ; \gamma ; z))
$$

to show that $\Re e(p(z))>0$ for all $z \in \mathbb{U}$.

It is clear that the function $\omega(z)$ both has the series form given by (1.6) and is analytic in the open set $\mathbb{U}$ of the complex plane. Therefore, the function $p(z)$ is also satisfies the conditions $p(0)=1$ and $n=1$, accentuated in Lemma 2.1.

It easily follows from (2.7) that

$$
\frac{d}{d z}(p(z))=\frac{d \omega}{d z}
$$

and in consideration of the equation determined in (2.4), the following relationships:

$$
\begin{aligned}
(1-z) z^{2} \frac{d^{3} \omega}{d z^{3}}+z[1+\gamma-(3+\alpha+\beta) z] & \frac{d^{2} \omega}{d z^{2}} \\
-(1+\alpha+\beta) z \frac{d \omega}{d z} & =\alpha \beta z \omega^{\prime}(z) \\
& \equiv \alpha \beta \Phi(z) \quad \text { (say) }
\end{aligned}
$$

is easily identified, where $z \in \mathbb{U}, \alpha \in \mathbb{C}-\mathbb{Z}_{0}^{-}$and $\beta \in \mathbb{C}-\mathbb{Z}_{0}^{-}$.

Suppose now that the related function $p(z)$ is not with positive real part in the domain $\mathbb{U}$. In the circumstances, under the conditions (2.2) and (2.3) of Lemma 2.1, there is a point $z_{0} \in \mathbb{U}$ such that

$$
\left.p(z)\right|_{z=z_{0}}=p\left(z_{0}\right)=i \lambda \quad \text { and }\left.\quad z \frac{d}{d z}[p(z)]\right|_{z=z_{0}}=\mu
$$

where

$$
n=1, \quad \lambda \in \mathbb{R}-\{0\} \quad, \quad \mu \in \mathbb{R} \quad \text { and } \mu \leq-\frac{1+\lambda^{2}}{2} .
$$

Then, under favour of the assumptions above, from (2.9), it follows that

$$
\Re e\left(\alpha \beta \Phi\left(z_{0}\right)\right)=\mu \Re e(\alpha \beta)\left\{\begin{array}{lll}
\leq-\frac{\Re e(\alpha \beta)}{2} & \text { if } & \Re e(\alpha \beta) \geq 0 \\
\geq-\frac{\Re e(\alpha \beta)}{2} & \text { if } \quad \Re e(\alpha \beta) \leq 0
\end{array},\right.
$$

where

$$
\alpha \in \mathbb{C}-\mathbb{Z}_{0}^{-} \quad, \quad \beta \in \mathbb{C}-\mathbb{Z}_{0}^{-} \quad \text { and } \quad \mu \leq-\frac{1+\lambda^{2}}{2} \quad(\lambda \in \mathbb{R}-\{0\}) .
$$


But, these cases above are contradictions, respectively, with the cases of the inequality, given in (2.5). Therefore, the function $p(z)$, defined by (2.7), immediately yields the the inequality given by (2.6). So, this completes the proof of Theorem 2.1.

As we indicated in the first section, the function $F(\alpha, \beta ; \gamma ; z)$ defined by $(1.1)$ plays very important roles in mathematical analysis and its applications. Specially, it also enables us to solve many important and interesting problems, such as conformal mapping of triangular domains bounded by line segments or circular arcs and various problems of quantum mechanics. Most of the functions that occur during analysis (or searches) can be expressed with nearly special forms of the hypergeometric functions.

In order to emphasize the importance of our main result, we here think useful to provide some information again. Especially, the series representation in (1.1) gives researchers much more motivations for their investigations; that is, the fact that the elementary functions and several other important functions in mathematics can be stated in terms of hypergeometric functions. Moreover, hypergeometric functions can be described as solutions of special second order linear differential equations that we pointed out as the hypergeometric differential equations given as in (1.6). Afterwards, Riemann was the first to raise this idea and introduce a special symbol to classify hypergeometric functions by singularities and exponents of differential equations. As we have also noted in the section 1, the hypergeometric function is a solution of the hypergeometric differential equation given in (1.6). The generalization of this equation to three arbitrary regular singular points is given by Riemanns differential equation. Any second order differential equation with three regular singular points can be transformed to the hypergeometric differential equation by changing of its variable. For more information, see the works given by the references in [2], [5]-[6], [9], [10], [16], [17], [20], [23], [31], [32], [38]-[41] and [43]-[51]. Therefore, as a requirement of the above explanations, in view of the above those relationships between the function given in (1.1) and the equation given in (1.6) will be important for our novel investigation. For this reason, our main result and their implications have various novel and/or nonlinear relations between them. Moreover, the desired research can be further expanded, taking into consideration the derivatives mentioned in (1.4) (or (1.5) for all the possible results that can be obtained. Accordingly, to determine all those results will be determined by the related elementary and also special functions, we need first recall some extra information in relation with the related definitions in (1.1) and (1.6), which are in the following forms.

(i) Some of Elementary Functions:

$$
\begin{gathered}
(1+z)^{n}=F(-n, 1 ; 1 ;-z) \quad(z \in \mathbb{C}), \\
\frac{1}{1-z}=F(1,1 ; 1 ; z) \quad(z \in \mathbb{C}-\{0\}), \\
\cos (z)=F\left(1 / 2,-1 / 2 ; 1 / 1 ; \sin ^{2} z\right) \quad(z \in \mathbb{C}), \\
\ln (1+z)=z F(1,1 ; 2 ;-z) \quad(z \in \mathbb{C}-\{-1\}), \\
\ln \left(\frac{1+z}{1-z}\right)=2 z F\left(1 / 2,1 ; 3 / 2 ; z^{2}\right) \quad(z \in \mathbb{C}-\{ \pm 1\}),
\end{gathered}
$$


The hypergeometric equations and the hypergeometric functions

$$
\begin{gathered}
\exp (z)=\lim _{\beta \rightarrow \infty} F(1, \beta ; 1 ; z / \beta) \quad(z \in \mathbb{C}), \\
\arcsin (z)=z F\left(1 / 2,1 / 2 ; 3 / 2 ; z^{2}\right) \quad(z \in \mathbb{C})
\end{gathered}
$$

and

$$
\arctan (z)=z F\left(1 / 2,1 ; 3 / 2 ;-z^{2}\right) \quad(z \in \mathbb{C}) .
$$

(ii) The Complete Elliptic Integrals of the First and Second Kinds:

$$
K(z)=\frac{\pi}{2} F\left(1 / 2,1 / 2 ; 1 ;-z^{2}\right) \quad(z \in \mathbb{C})
$$

and

$$
E(z)=\frac{\pi}{2} F\left(-1 / 2,1 / 2 ; 1 ;-z^{2}\right) \quad(z \in \mathbb{C})
$$

(iii) The Adjoint Legendre Functions:

$$
\begin{gathered}
P_{m}^{n}(z)=\frac{(z+1)^{n / 2}}{(z-1)^{m / 2}} \frac{1}{\Gamma(1-n)} F\left(-n, n+1 ; 1-m ; \frac{1-z}{2}\right) . \\
\left(-n, m \in \mathbb{N}_{0} ; z \in \mathbb{C}\right)
\end{gathered}
$$

(iv) The Chebyshev Polynomials:

$$
T_{n}(z)=F\left(-n, n ; \frac{1}{2} ; \frac{1-z}{2}\right) \quad\left(n \in \mathbb{N}_{0} ; z \in \mathbb{C}\right) .
$$

(v) The Legendre Polynomials:

$$
P_{n}(z)=F\left(-n, n+1 ; 1 ; \frac{1-z}{2}\right) \quad\left(n \in \mathbb{N}_{0} ; z \in \mathbb{C}\right) .
$$

(vi) The Gegenbauer (Ultraspherical) Polynomials:

$$
\begin{gathered}
C_{n}^{\alpha}(z)=\frac{(1+\alpha)_{n}}{\Gamma(n+1)} F\left(-n, n+2 \alpha ; \alpha+\frac{1}{2} ; \frac{1-z}{2}\right) \\
\left(n \in \mathbb{N}_{0} ; \alpha \in \mathbb{Z}_{0}^{-} ; z \in \mathbb{C}\right) .
\end{gathered}
$$

(vii) The Jacobi Polynomials:

$$
\begin{gathered}
P_{n}^{\alpha, \beta}(z)=\frac{(1+\alpha)_{n}}{\Gamma(n+1)} F\left(-n, 1+n+\alpha+\beta ; \alpha+1 ; \frac{1-z}{2}\right) . \\
\left(n \in \mathbb{N}_{0} ; \alpha \in \mathbb{Z}_{0}^{-} ; \beta \in \mathbb{Z}_{0}^{-} ; z \in \mathbb{C}\right) .
\end{gathered}
$$

(viii) The Confluent Hypergeometric Function ${ }_{1} F_{1}(\alpha ; \beta ; z)$ :

$$
{ }_{1} F_{1}(\alpha ; \beta ; z)=\lim _{\gamma \rightarrow \infty} F\left(\alpha, \gamma ; \beta ; \frac{z}{\gamma}\right) \quad\left(\alpha, \beta, \gamma \in \mathbb{Z}_{0}^{-} ; z \in \mathbb{C}\right) .
$$

(ix) The Error Functions:

$$
\operatorname{erf}(z)=\frac{2 z}{\sqrt{\pi}}{ }_{1} F_{1}\left(1 / 2 ; 3 / 2 ;-z^{2}\right) \quad(z \in \mathbb{C})
$$


and

$$
\operatorname{erfc}(z)=\frac{1}{\sqrt{\pi}} e^{-z^{2}}{ }_{1} F_{1}\left(1 / 2 ; 1 / 2 ; z^{2}\right) \quad(z \in \mathbb{C})
$$

and so on. For the details of those functions (or series representations), one may refer to the works given by the references in [2], [5], [15], [6], [12], [18], [20], [34], [38], [40] and [43]-[51].

As certain special implications of our main result, when considering the important relationships signified by (2.10)-(2.27), it is naturally easy to determine a number of special results, which are related to the main result, namely, Theorem 2.2. In order to determine both appropriate sampling and possible special results, we would like to leave the researchers with detailed research and emphasize only two of them, as examples.

As one of the special implications, we would like to point out a comprehensive result in relation with the confluent hypergeometric function that we mentioned as in (2.25). For this, we would like to remind researchers of some detailed information about this particular results again.

The following functional series with the complex variable $z$ :

$$
\begin{aligned}
{ }_{1} F_{1}(\alpha ; \beta ; z) & =1+\frac{\alpha}{\beta} z+\frac{\alpha(\alpha+1)}{\beta(\beta+1)} \frac{z^{2}}{2 !}+\ldots \\
& =\sum_{k=0}^{\infty} \frac{(\alpha)_{k}}{(\beta)_{k}} \frac{z^{k}}{k !} \\
(z \in \mathbb{U} ; \alpha & \left.\in \mathbb{C}-\mathbb{Z}_{0}^{-} ; \beta \in \mathbb{C}-\mathbb{Z}_{0}^{-}\right),
\end{aligned}
$$

where $(\alpha)_{k}$ and $(\beta)_{k}$ are the Pochhammer symbols defined by (1.2), in generally, is called as the confluent hypergeometric function in the literature. Clearly, it defines an analytic function for all finite $z$, is closely connected the hypergeometric function given by (1.1), and is then obtained as a limit of $F(\alpha, \beta ; \gamma ; z / \beta)$ when $\beta$ tends to $\infty$ as it was indicated in (2.25). It is clear that the confluent hypergeometric function is a degenerate form of the hypergeometric function ${ }_{2} F_{1}(\alpha ; \beta ; \gamma ; z)$ which arises as a solution of the confluent hypergeometric differential equation given by above.

Since the confluent hypergeometric function is any of the solutions of the following second-order ordinary linear differential equation:

$$
\begin{gathered}
z \frac{d^{2} \omega}{d z^{2}}+(\alpha-z) \frac{d \omega}{d z}-\beta \omega=0 \\
\left(z \in \mathbb{C} ; \alpha \in \mathbb{C}-\mathbb{Z}_{0}^{-} ; \beta \in \mathbb{C}-\mathbb{Z}_{0}^{-}\right),
\end{gathered}
$$

this differential equation is also called as the confluent hypergeometric differential equation in the literature.

The first special implication of our main result is contained in the following proposition below. 
Proposition 2.3. Let the function $\omega:=\omega(z)$ be in the form given as (2.28) and also let any one of the cases of the following inequality:

$$
\begin{gathered}
\Re e\left\{z^{2} \frac{d^{3} \omega}{d z^{3}}+[1+(\alpha-z)] z \frac{d^{2} \omega}{d z^{2}}-z \frac{d \omega}{d z}\right\} \\
\left\{\begin{array}{l}
>-\frac{\Re e(\beta)}{2} \quad \text { if } \quad \Re e(\beta) \geq 0 \\
<-\frac{\Re e(\beta)}{2} \quad \text { if } \quad \Re e(\beta) \leq 0
\end{array}\right.
\end{gathered}
$$

be provided for any $z \in \mathbb{U}$ and for some $\alpha \in \mathbb{C}-\mathbb{Z}_{0}^{-}$and $\beta \in \mathbb{C}-\mathbb{Z}_{0}^{-}$. In the present case,

is also provided.

$$
\Re e(\omega(z))>0 \quad(z \in \mathbb{U})
$$

Proof. By means of the information presented as in (2.28) and (2.29), and also in consideration of the proof of Theorem 2.2 , if one takes the function $p(z)$, defined as in (2.7), namely, define it in the form:

$$
\begin{gathered}
p(z)=\omega(z)\left(\equiv{ }_{1} F_{1}(\alpha ; \beta ; z)\right) \\
\left(z \in \mathbb{U} ; \alpha \in \mathbb{C}-\mathbb{Z}_{0}^{-} ; \beta \in \mathbb{C}-\mathbb{Z}_{0}^{-}\right),
\end{gathered}
$$

and then the related steps used (in the proof of Theorem 2.2) are again followed, the desired proof can be easily obtained. Here, its details are left to the researchers.

Through the instrument of the relation between the hypergeometric function and the complex error function in (2.26) together with (2.27), as second implication of our main result, certain special results can be also obtained between the various inequalities associated with error functions in the complex plane. For those, in (2.26), (2.28) and (2.29), respectively, by choosing the suitable values of the parameters $\alpha$ and $\beta$, one can derive some of them. For example, by setting

$$
\alpha:=\frac{1}{2} \quad \text { and } \quad \beta:=\frac{3}{2}
$$

in (2.26), the following results:

$$
\begin{gathered}
{ }_{1} F_{1}\left(1 / 2 ; 3 / 2 ;-z^{2}\right)=\frac{\sqrt{\pi}}{2} \frac{\operatorname{erf}(z)}{z} \\
(z \in \mathbb{D}:=\mathbb{U}-\{0\}), \\
{ }_{1} F_{1}(1 / 2 ; 3 / 2 ; z)=2 \sum_{k=0}^{\infty} \frac{(-z)^{k}}{k !} \quad(z \in \mathbb{U})
\end{gathered}
$$

and

are easily obtained.

$$
2 z \frac{d^{2} \omega}{d z^{2}}+(1-2 z) \frac{d \omega}{d z}-3 \omega=0 \quad(z \in \mathbb{U})
$$

So, as we have informed above, the following-special function:

$$
{ }_{1} F_{1}\left(1 / 2 ; 3 / 2 ;-z^{2}\right) \quad(z \in \mathbb{U}),
$$


which is given by (2.32) (or (2.33)), is a solution for the second-order linear differential equation given by (2.34). After these explanations, the following proposition, i.e., Proposition 2.4 below, can be easily proved within the scope of the rationale of the main result (or Proposition 2.3). The detail of the related proof has been left to the researchers again.

Proposition 2.4. For any $z \in \mathbb{D}($ or, $z \in \mathbb{U})$, if the inequality:

$$
\Re e\left\{2 z^{2} \frac{d^{3}}{d z^{3}}\left(\frac{\operatorname{erf}(z)}{z}\right)+[2+(1-2 z)] z \frac{d^{2}}{d z^{2}}\left(\frac{\operatorname{erf}(z)}{z}\right)-2 z \frac{d}{d z}\left(\frac{\operatorname{erf}(z)}{z}\right)\right\}>-\frac{3}{\sqrt{\pi}}
$$

is ensured, then

$$
\Re e\left(\frac{\operatorname{erf}(z)}{z}\right)>0
$$

is also ensured.

\section{References}

[1] Abad, J., Sesma, J., Buchholz polynomials: A family of polynomials relating solutions of confluent hypergeometric and Bessel equations, J. Comput. Appl. Math., 101(1999), 237-241.

[2] Abramowitz, M., Stegun, I.A., Confluent Hypergeometric Functions in Handbook of Mathematical Functions with Formulas, Graphs and Mathematical Tables, New York, 1972.

[3] Ancarani, L.U., Gasaneo, G., Derivatives of any order of the confluent hypergeometric function $_{1} F_{1}(a, b, z)$ with respect to the parameter $a$ or $b$, J. Math. Phys., 49(2008), no. $6,16 \mathrm{pp}$.

[4] Anderson, G.D., Vamanamurthy, M.K., Vuorinen, M., Conformal Invariants, Inequalities and Quasiconformal Maps, John Wiley \& Sons, 1997.

[5] Andrews, G.E., Askey, R., Roy, R., Special Functions, Vol. 71 of Mathematics and its Applications, Cambridge University Press, UK, 1999.

[6] Bailey, W.N., Generalized Hypergeometric Series, Cambridge University Press, Cambridge, 1935, Reprinted: Stechert-Hafner, New York, 1964.

[7] Badralexe, R., Marksteiner, P., Oh, Y., Freeman, A.J., Computation of the Kummer functions and Whittaker functions by using Neumann type series expansions, Comput. Phys. Commun., 71(1992), 47-55.

[8] Balasubramanian, R., Ponnusamy, S., Vuorinen, M., On hypergeometric functions and function spaces, J. Comput. Appl. Math., 139(2002), 299-322.

[9] Bell, W.W., Special Functions for Scientists and Engineers, D. Van Nostrand Company Ltd., Reinhold, New York, 1968.

[10] Carlson, B., Special Functions of Applied Mathematics, Academic Press, New York, 1977.

[11] Chiarella, C., Reichel, A., On the evaluation of integrals related to the error function, Math. Comp., 22(1968), 137-143.

[12] Coffey, M.W., Johnston, S.J., Some results involving series representations of hypergeometric functions, J. Comp. Appl. Math., 233(2009), 674-679. 
[13] Dotsenko, M., On some applications of Wright's hypergeometric function, C.R. Acad. Bulgare Sci., 44(1991), 13-16.

[14] Dunster, T.M., Asymptotic approximations for the Jacobi and ultraspherical polynomials and related functions, Methods Appl. Anal., 6(1999), 21-56.

[15] El-Attar, R., Special Functions and Orthogonal Polynomials, Lulu Press, USA, 2006.

[16] Erdelyi, A., Mangus, W., Oberhettinger, F., Tricomi, F.G., Higher Transcendental Functions, Vol. I, McGraw-Hill, New York, 1953.

[17] Farrell, O.J., Ross, B., Solved Problems in Analysis, as applied to Gamma, Beta, Legendre and Bessel Functions, Dover Publications, New York, 1971.

[18] Ferreira, C., Lopez, J.L., Sinusia, E.P., The Gauss hypergeometric function $F(a, b ; c ; z)$ for large c, J. Comput. Appl. Math., 197(2006), 568-577.

[19] Forrey, R.C., Computing the hypergeometric function, J. Comput. Phys., 137(1997), 79100.

[20] Gasper, G., Rahman, M., Basic Hypergeometric Series, Encyclopedia of Mathematics and its Applications, Cambridge University Press, Cambridge, UK, 1990.

[21] Gautschi, W., Efficient computation of the complex error function, SIAM J. Numer. Anal., 7(1970), 187-198.

[22] Gil, A., Segura, J., Temme, N.M., Numerical Methods for Special Functions, Society for Industrial and Applied Mathematics, 2007.

[23] Gradshteyn, I.S., Ryzhik, I.M., Table of Integrals, Series and Products, 7th ed. Elsevier, Academic Press, Amsterdam, 2007.

[24] Hale, N., Townsend, A., Fast and accurate computation of Gauss-Legendre and GaussJacobi quadrature nodes and weights, SIAM J. Sci. Comput., 35(2013), 652-674.

[25] Hsu, Y.P., Development of a Gaussian hypergeometric function code in complex domains, Int. J. Modern Phys., 4(1993), 805-840.

[26] Irmak, H., Certain complex equations and some of their implications in relation with normalized analytic functions, Filomat, 30(2016), 3371-3376.

[27] Irmak, H., Some novel applications in relation with certain equations and inequalities in the complex plane, Math. Commun., 23(2018), 9-14.

[28] Irmak, H., Agarwal, P., Some comprehensive inequalities consisting of Mittag-Leffler type functions in the complex plane, Math. Model. Nat. Phenom., 12(2017), 65-71.

[29] Irmak, H., Bulboacă, T., Tuneski, N., Some relations between certain classes consisting of $\alpha$-convex type and Bazilevic type functions, Appl. Math. Lett., 24(2011), 2010-2014.

[30] Kalla, S.L., On the evaluation of the Gauss hypergeometric function, C.R. Acad. Bulgare Sci., 45(1992), 35-36.

[31] Kanemitsu, S., Tsukada, H., Vistas of Special Functions, World Scientific Publishing Company, Singapore, 2007.

[32] Lebedev, N.N., Special Functions and their Applications, Prentice Hall, New Jersey, 1965.

[33] McKenna, S.J., A method of computing the complex probability function and other related functions over the whole complex plane, Astrophys. Space Sci., 107(1984), 71-83.

[34] Morita, T., Use of the gauss contiguous relations in computing the hypergeometric functions ${ }_{2} F_{1}(n+1 / 2, n+1 / 2 ; m ; z)$, Interdiscip. Infor. Sci., 2(1996), 63-74.

[35] Nunokawa, M., Goyal, S.P., Kumar, R., Sufficient conditions for starlikeness, J. Class. Anal., 1(2012), 85-90. 
[36] Nunokawa, M., Sokol, J., Thomas, D., On Ozaki's condition for p-valency, C.R. Math. Acad. Sci. Paris, 356(2018), 382-386.

[37] Olver, F.W.J., Asymptotics and Special Functions, AKP Classics, A.K. Peters Ltd., Wellesley, MA, 1997.

[38] Olver, F.W.J., Lozier, D.W., Boisvert, R.F., Clark, C.W., NIST Handbook of Mathematical Functions, Cambridge University Press, New York, USA, 2010.

[39] Ponnusamy, S., Hypergeometric transforms of functions with derivative in a half plane, J. Comput. Appl. Math., 96(1998), 35-49.

[40] Rainville, E.D., Special Functions, The Macmillan, New York, NY, USA, 1960.

[41] Rainville, E.D., Special Functions, Chelsea Publishing Company, New York, 1960.

[42] Rakha, M.A., El-Sedy, E.S., Application of basic hypergeometric series, Appl. Math. Comput., 148(2004), 717-723.

[43] Seaborn, J.B., Hypergeometric Functions and their Applications, Springer-Verlag, 1991.

[44] Slater, L.J., Confluent Hypergeometric Functions, Cambridge University Press, 1960.

[45] Slater, L.J., The Second Form of Solutions of Kummer's Equations and Confluent Hypergeometric Functions, Cambridge, Cambridge University Press, 1960.

[46] Slater, L.J., Generalized Hypergeometric Functions, Cambridge University Press, 1966.

[47] Şan, M., Irmak, H., Some novel applications of certain higher order ordinary complex differential equations to normalized analytic functions, J. Appl. Anal. Comput., 5(2015), 479-484.

[48] Temme, N.M., Special Functions: An Introduction to the Classical Functions of Mathematical Physics, Wiley, 1996.

[49] Wallman, H., Transient response and the central limit theorem of probability, Proc. Symposia Appl. Math., 2(1950), 91-112.

[50] Wang, Z.X., Guo, D.R., Special Functions, World Scientific, Singapore, 2010.

[51] Zhang, S., Jin, J., Computation of Special Functions, Wiley, 1966.

Hüseyin Irmak

Çankırı Karatekin University,

Faculty of Science, Department of Mathematics,

Uluyazı Campus, TR 18100, Çankırı, Turkey

e-mail: hirmak@karatekin.edu.tr, hisimya@yahoo.com 